\section{Post-modern synthesis?}

\author{
Developmental plasticity and evolution \\ Mary Jane West-Eberhard \\ Oxford University Press, New York; 2003. 794 pp. \\ Price £37.50, paperback. ISBN 0-19-5122356
}

Heredity (2004) 92, 596-597. doi:10.1038/sj.hdy.6800471

\section{Reviewed by PC Luttikhuizen and J Drent}

Development was left out of the Modern Synthesis of evolutionary biology. As with a sulky child not joining a game, it is not clear whether the others did not want it or whether it did not want them. Fortunately, there is such a thing as generations, and in the new generation the sulkiness may have been diluted. Many now believe the time is ripe for a reconciliation - evidenced by the rapidly growing branch called 'evo-devo'. The hand that is extended from the side of the Modern Synthesis toward development is phenotypic plasticity, or the map between the genotype and the phenotype. West-Eberhard's new book is one of the most extensive efforts to bring together what is known and suspected of the nature and consequences of this map.

In 1989, West-Eberhard wrote a review on the influence of the phenotype and its plasticity on the course of evolution. In it, she emphasized the very true, to most obvious, but in the era of genetics arguably much ignored point, that selection is on the phenotype, and that between the genotype and the phenotype lies a trajectory that includes development, parental effects and adaptive plasticity (West-Eberhard, 1989).

In 1989, she wrote 'When exploited more thoroughly and more expertly than is possible for one person in one review, attention to the nature of the phenotype will clarify many issues (eg regarding models of speciation, social traits, and the origin of major novelties) sometimes considered 'difficulties' for theories of evolution that neglect plasticity'. The fact that she devoted many years to writing the current single-authored, almost 800-page volume on the subject perhaps indicates that she has not been altogether satisfied with the amount and nature of the attention that has been given to plasticity in the previous decade. This is evidenced also from some chilly criticisms in the book.

The book is divided into four parts. The first describes the main thesis of the book, essentially unchanged since the 1989 review: the attempt to make a synthesis between genetic variation, environmental influences on phenotypes, developmental effects and evolution. Environmental responsiveness of organisms is universal and influences (together with genes) individual development and organic evolution. Though phenotypic plasticity has entered the thinking of most biologists today, the restricted view that this plasticity makes genetic variation invisible to selection, or that it is not more than an organismal trait for which genetic variation may exist, still thrives. Most would not ascribe to phenotypic plasticity the prominent role West-Eberhard would have it play. She advocates the idea that the phenotypic variation available to selection is to a large extent the product of developmental plasticity and flexibility, and that, because of this, the environment moulding the phenotype is of greater influence on the course and outcome of evolution than usually appreciated. She uses the term 'genetic accommodation', which is meant to be a broader version of what is known as 'genetic assimilation' or the 'Baldwin effect'.

Like plasticity, the canalized nature of development could be seen as a compensating effect simply obscuring mutational variation from selection. However, WestEberhard points out that correlated shifts in related traits in response to an environmental or mutational change could also magnify the effect of such a change. A refreshing description is given of the time dependence of phenotypes, which she calls 'continuity of the phenotype': the state of the phenotype not only depends on current environment and current genetics, but also on the environments experienced in the (developmental) history of the individual, and possibly also the genetics of its parents and their environmental history.

The many ways in which novelties in phenotype could arise are treated in the second part. Expanding on the usual deletion, duplication and insertion, she discusses how parts of morphology and behaviour can change in similar ways. This is probably the part in the book where development blooms best; non-evo-devoists learn here how changes in timing or location of gene expression and alternative RNA splicing can produce novel phenotypes, and how recurrent anomalies are potential sources of eventually advantageous variants.

The third part is a review of alternative phenotypes, which is what the author set out to write a book on, until she decided in favour of the larger exercise. Alternative phenotypes are here defined as the result of genetic differences (allelic-switch control) or environmental influence (condition-dependent expression). The questions, 'how common are they?' and 'are complex morphological traits?' more often condition-dependent than simple ones are only qualitatively answered. The interweaving of the several subjects (the evidence for alternative phenotypes, is their nature allelic-switch, condition-dependent or a complex combination, and is the variation maintained in stability or dynamically) seems to us to somewhat blur the separate points made. This could, admittedly, be caused by the fact that very little work has been carried out in this field, both empirically and theoretically, although some of the more recent literature is lacking from the book.

The last part shifting from the role of the genotype to an integrated role for environment, development and genotype, discusses what the proposed difference in emphasis, implies for our views of major themes in evolution, such as speciation.

Readers with a mathematical inclination will find the theoretical support wanting. Not only does West-Eberhard herself not translate verbal models into mathematical ones, she also does not use those made by others. However simplified traditional quantitative genetics models may be, they need not be discarded without further thought, but could instead be put to good use as 
the backbone for any innovations. As she herself recognizes, most of the ideas in the book will have to be explored for soundness using mathematical models, and, in fact, this is already happening (eg Stadler et al, 2001; Garson et al, 2003).

The book is not an easy read. The writing is sometimes difficult to follow and can be confusing in its structure. Chapters and sections often appeared to us to be organized inside-out or upside-down; the author will start with an abstract description of what she is trying to say, while the specific examples that are the basis for the conclusions as well as explicit definitions of terms used come later. The amount of referring back and forth among the sections of the book and the use of brackets are quite extensive and do not improve readability.

In summary, these ideas, weaved together in this book, are not new, as the author herself stresses. However, they are presented with a strongly shifted emphasis. The importance of the subject should inspire many biologists to see through the charging criticism and difficult style of writing. Whether it is possible to arrive at a post-Modern Synthesis, as the author proposes we should, we will not know unless we try. West-Eberhard claimed in 1989 that her present effort would be too much for one person; hopefully, her book will be a stimulating appetizer for

\section{References}

Garson J, Wang L, Sarkar S (2003). How development may direct evolution. Biol Philos 18: 353-370.

Stadler BMR, Stadler PF, Wagner GP, Fontana W (2001). The topology of the possible: formal spaces underlying patterns of evolutionary change. I Theor Biol 213: 241-274.

West-Eberhard MJ (1989). Phenotypic plasticity and the origins of diversity. Annu Rev Ecol System 20: 249-278.

PC Luttikhuizen ${ }^{1}$ and J Drent ${ }^{2}$ ${ }^{1}$ Institute for Biodiversity and Ecosystems, University of Amsterdam, Experimental Plant Systematics, Amsterdam, The Netherlands, ${ }^{2}$ Department of Marine Ecology and Evolution, Royal Netherlands Institute for Sea Research, Den Burg/University of Groningen, Centre for Ecological and Evolutionary Studies, Haren, The Netherlands

E-mail: luttikhuizen@science.uva.nl 